\title{
Clinical and histopathological activity in paucibacillary leprosy patients after fixed-duration multidrug therapy
}

\author{
G. J. EBENEZER, S. SUNEETHA \& S. ARUNTHATHI \\ Department of Histopathology, Schieffelin Leprosy Research and \\ Training Centre, Karigiri, North Arcot Ambedkar District, Tamil \\ Nadu, India 632106
}

Accepted for publication 27 March 1997

Summary In 37 clinically-diagnosed borderline-tuberculoid (BT) leprosy patients skin biopsies were done prior to starting multidrug therapy (MDT) and at the end of 6 months therapy. Clinical and histopathological activity, graded as active, resolving and inactive, were studied at the end of 6 months of MDT.

Of the 37 clinically-diagnosed BT patients 24 could be confirmed by histopathology as having BT leprosy, while the other 13 biopsies showed features of indeterminate (I) leprosy. After 6 months of MDT, out of the 24 histopathologically-confirmed BT patients, $4(17 \%)$ showed clinical activity and $8(33 \%)$ showed histopathological activity. Of the 13 histopathologically-diagnosed indeterminate cases all were clinically inactive but histological activity persisted in 3 cases (23\%). Out of the 37 clinicallydiagnosed BT patients 3 showed both clinical and histopathological activity at the end of MDT.

This study emphasizes the importance of performing histopathological examinations on leprosy patients undergoing research studies for the confirmation of diagnosis and for proper classification of the disease. The histopathological activity that outlasts the MDT may be due to the bacillary fragments that persist but clinical activity coupled with histopathological activity seen in 3 patients at the end of 6 months may foreshadow a relapse and these patients and others like them need to be followed up for longer durations.

\section{Introduction}

The World Health Organization (WHO) study group on chemotherapy of leprosy for control programmes had classified leprosy patients into multibacillary (MB) and paucibacillary $(\mathrm{PB})$ groups. In 1982, the PB group included all polar tuberculoid (TT), borderline tuberculoid (BT) and indeterminate (I) cases diagnosed clinically or histopathologically with a BI of 2 or less than 2 on the Ridley scale. ${ }^{1}$ The MB group consists of polar lepromatous (LL), borderline lepromatous (BL) and mid-borderline (BB) patients. In 1988 this was modified again and all smear positive patients were included in the MB group for treatment with multidrug therapy (MDT). ${ }^{2}$ The paucibacillary type of leprosy constitute $70 \%$ of the total number of leprosy 
patients in India. ${ }^{3}$ It has been observed that a significant number of patients present with a single lesion. These lesions may heal or progress towards the multibacillary part of the spectrum. WHO has recommended a fixed duration of MDT for PB patients, i.e. dapsone $100 \mathrm{mg}$ daily and rifampicin $600 \mathrm{mg}$ once a month for 6 months. The short duration of treatment and relapses in PB cases raises a few basic issues regarding the clinical activity and the histopathological changes which occur in patients on PB-MDT. This study has been carried out to elucidate such changes.

\section{Materials and methods}

This is a study of 37 leprosy patients who had been clinically diagnosed and classified according to the Ridley-Jopling classification ${ }^{4}$ as BT patients, attending the Outpatient Department at the Schieffelin Leprosy Research and Training centre, Karigiri, S. India. Each patient had a complete clinical examination done, recording the morphology and the site of lesions. Skin-smear examination for acid-fast bacilli was done from routine and selective sites and were negative in all 37 patients. The characteristic clinical features of skin lesions of patients being categorized as belonging to the BT group included single or multiple, dry, hypopigmented, flat or raised patches of any size with ill-defined or well-defined margins. Modalities of sensation were lost to varying degrees in these patches. None of the patients had nerve trunk lesions.

A biopsy of skin lesion was taken before starting MDT. An elliptical piece of skin was biopsied from an active patch under local anaesthesia by the standard Khanolkar technique. ${ }^{5}$ The skin biopsies were fixed in formol zenker. Serial sections of $5 \mu$ thickness were stained with haematoxylin and eosin for routine study and a modified Fite-Faraco stain for acid-fast bacilli. ${ }^{6}$ Patients were diagnosed and classified histopathologically according to the classification given by Ridley \& Jopling. ${ }^{4}$ Histologically, the lesions were classified as indeterminate leprosy when there was mild lymphocytic infiltration around skin adnexa and selective peri and intraneural infiltration with lymphocytes of dermal nerves. ${ }^{17}$ All patients were reassessed clinically and histologically at the end of 6 months of MDT. Skin bopsies were taken from the same skin lesion but at a different edge. Clinical assessment was recorded as follows: 'active' when erythema or infiltration persisted, a new lesion appeared, anaesthetic areas increased and or any nerve trunks became tender; 'resolving' when there was a decrease in infiltration, anaesthesia and size of the patch; 'inactive' when the patches were without infiltration and erythema.

The skin bopsies were assessed for the type of granuloma, granuloma fraction, nerve inflammation and for the presence of acid-fast bacilli (AFB). The histological findings were graded as 'active' when there was dermal infiltration by epithelioid granuloma and the granuloma fraction was more than $10 \%$ in the dermal tissue and nerve inflammation. It was graded as 'resolving' when the granuloma fraction was less than $10 \%$ and 'inactive' when epithelioid cell granuloma was absent and/or the lymphocytic infiltrate was less than $5 \%$.

\section{Results}

Of the 37 clinically-diagnosed BT patients, 27 were males and 10 were females. The age of the patients ranged from 7 to 50 years with a mean of $15 \cdot 5$ years. Out of the 37 cases, 24 


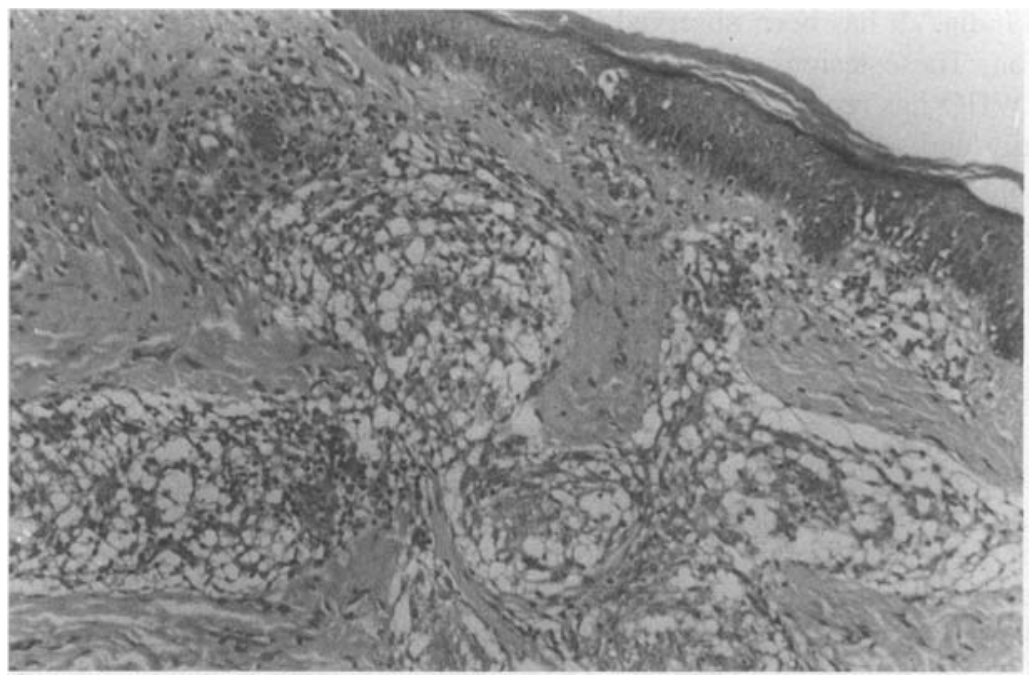

Figure 1. Photomicrograph to show tuberculoid granuloma composed of epithelioid cells, Langhan giant cells and lymphocytes infiltrating and replacing extensive areas of the dermis (active). $\mathrm{H} \& \mathrm{E} \times 100$.

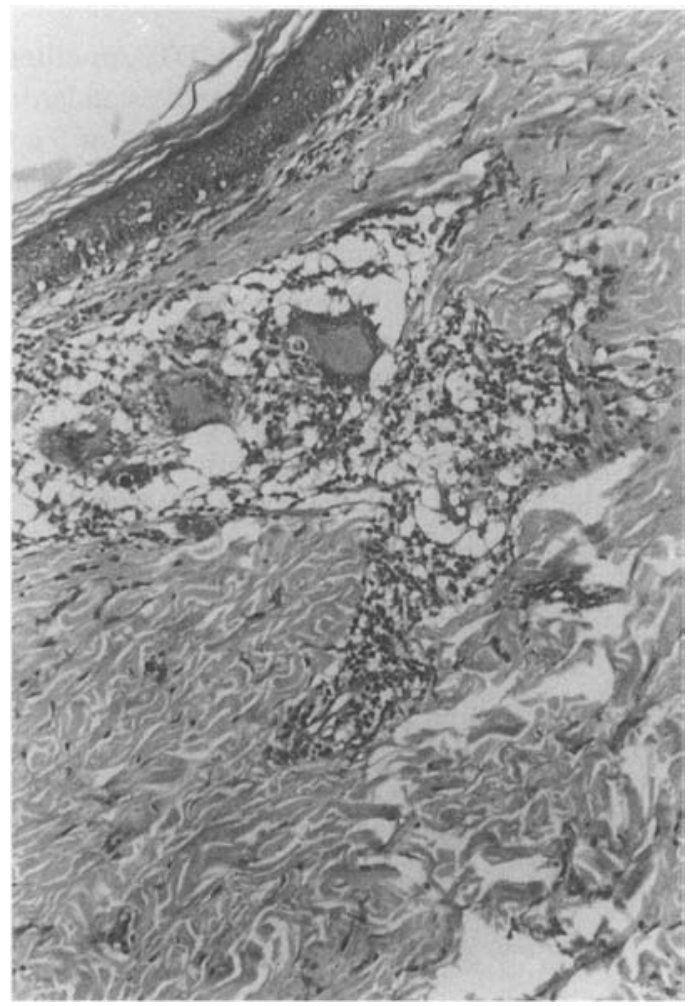

Figure 2. Resolving granuloma composed of a few Langhan giant cells in the small collection of epithelioid cells and lymphocytes (resolving). $\mathrm{H} \& \mathrm{E} \times 100$. 


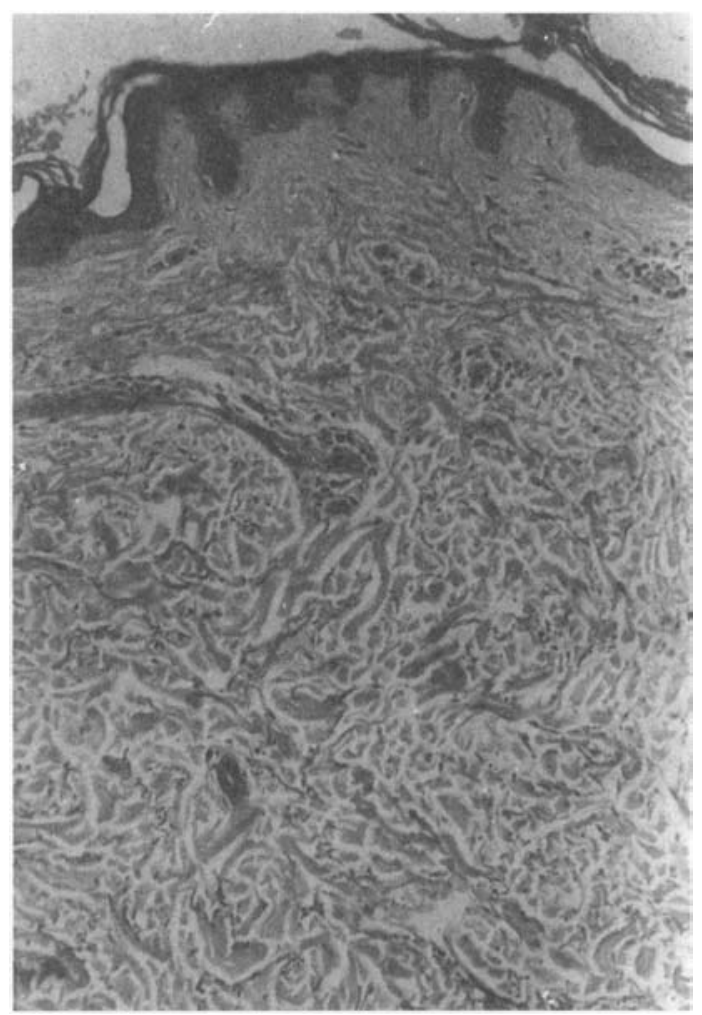

Figure 3. Photomicrograph to show a few scattered lymphocytes around blood vessels. Epithelioid granulomas are not seen (inactive). $\mathrm{H} \& \mathrm{E} \times 100$.

patients presented with single lesion and 13 of them had multiple patches but less than 5 in number. In 28 cases the lesions presented only in the upper and lower limbs and in 9 patients the patches were generalized. Histopathological examination of these 37 clinically-diagnosed BT patients showed that 24 of them had a histology that correspondedwith the BT type of the disease and 3 of these showed bacilli in the dermal nerves. The other 13 cases showed histological features that fitted in with the indeterminate type of leprosy. None of them had AFB.

The second examination was conducted after 6 months of MDT therapy. Of the 24 patients clinically and histopathologically diagnosed as BT, 4 were clinically active and 8 showed histologically-active persistent epithelioid granulomas (Figure 1). Of the 13 histologically-confirmed indeterminate cases none showed active signs of the clinical disease but histologically the lesions were active in 3 cases. Histological features of resolving granulomas (Figure 2) were seen in $5 \mathrm{BT}$ cases and in 1 indeterminate case. Eleven BT and 9 indeterminate cases were found to be inactive, with either absence of epithelioid granulomas and/or the lymphocytic infiltrate was less than 5\% (Figure 3). The granuloma fractions of BT leprosy patients before and after MDT is contrasted in Figure 4 and of indeterminate patients in Figure 5. None of the patients showed evidence of neuritis and none of the biopsies showed evidence of reversal reaction (RR) or acid-fast bacilli. 


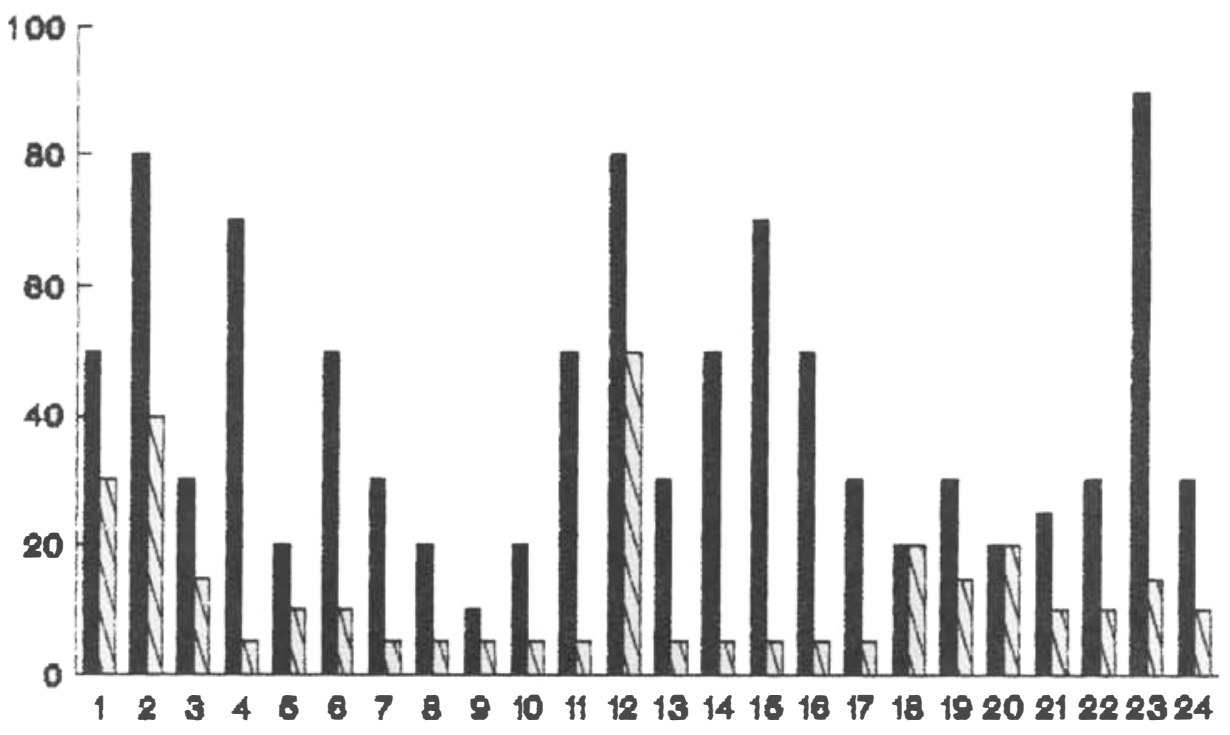

Figure 4. Granuloma fraction in BT leprosy.

\section{Discussion}

In this study, 37 patients were clinically diagnosed as BT following a precise categorization but on histopathological examination only 24 of them could be confirmed as having a BT histology while the other 13 showed features that were consistent with the indeterminate group of leprosy. Discrepancies have been reported by several authors between histopathological and clinical diagnosis of leprosy, ${ }^{7-9}$ and our finding again emphasizes the importance

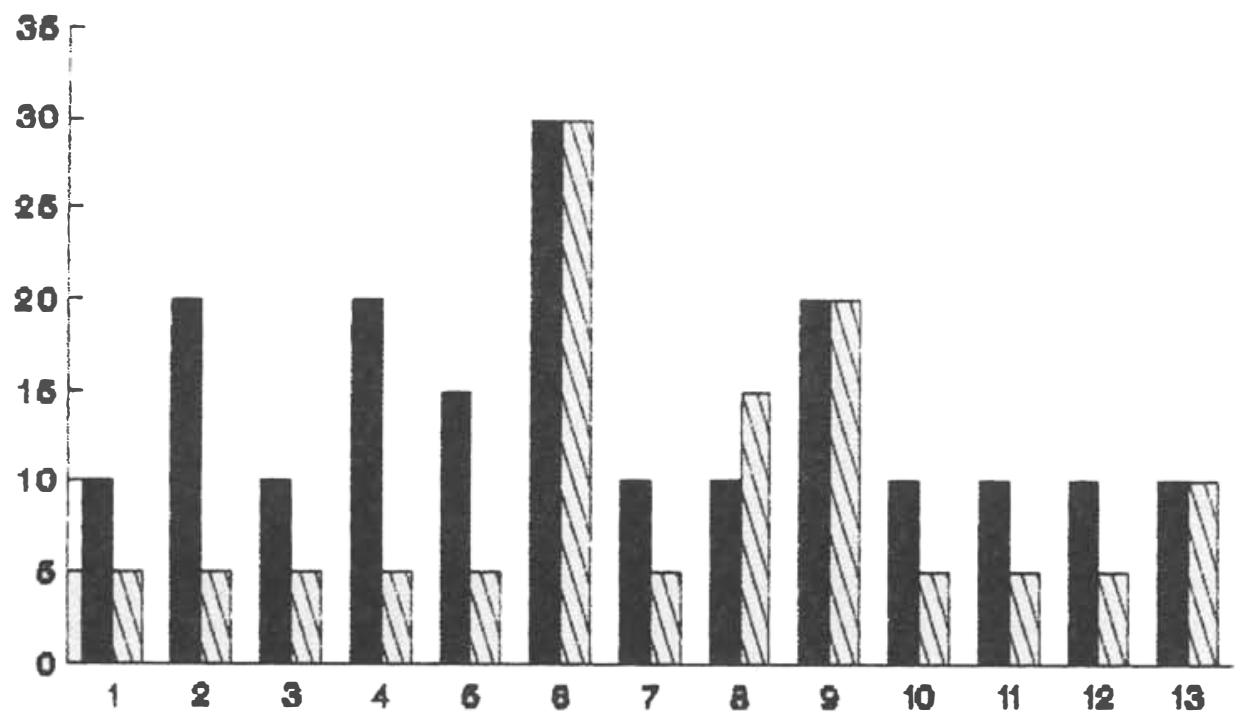

Figure 5. Granuloma fraction in indeterminate leprosy. 
of performing histopathological examinations on leprosy patients undergoing research studies for the confirmation of diagnosis and for proper classification of the disease.

MDT is prescribed for PB leprosy patients with an aim to reduce the duration of treatment and to deal with possible drug resistance to dapsone and to increase cost-effectiveness by releasing the patients from continuing the treatment at the point of inactivity. ${ }^{1}$ However persistence of disease activity has been observed in the skin lesions of the patients after 6 months of treatment. ${ }^{10-13}$ The reported percentage of PB patients who showed persistent activity at the end of 6 months of $\mathrm{PB}$ regimen ranges from $4.3 \%$ to $67 \%{ }^{14}$ The wide variations in the reported clinical response may be due to different concepts of classification and disease activity.

In our series of $24 \mathrm{BT}$ patients $4(17 \%)$ showed clinical activity and $8(33 \%)$ showed histopathological activity after completion of therapy. In 13 indeterminate cases all were clinically inactive but histologically the activity persisted in 3 cases (23\%) after 6 months of MDT therapy. The persistent activity in these patients may be the result of the continued presence of the antigens of the lepra bacilli. ${ }^{15,16}$ Evidence of resolution of the inflammatory lesion (5 in BT and 1 in indeterminate) would suggest that in due course, even the active inflammation would clear up as has been stated and that adequate immune response present in PB patient would be expected to remove these antigenic fragments.

However among the 11 histologically-active cases, 3 cases showed features of clinical activity too. Of the 11 histopathologically-active patients it is reasonable to expect a small percentage of these patients to continue to be active with a likelihood of a relapse occurring in due course. The relapse rate among PB patients has been reported to be as high as $12.9 \%{ }^{18}$ and $12 \%{ }^{19}$ and as low as $0 \cdot 34 \%{ }^{20}$ and $0 \cdot 5 \% .^{21}$ However WHO analysis of a large series of PB cases treated with MDT revealed the risk of relapse to be only $1.07 \%{ }^{22}$ Therefore a careful follow-up of the patients under report for a longer period is imperative. Sequential biopsies every year on a much larger number of PB patients, who had persistent active lesions at the end of 6 months of MDT, and a careful follow-up of these patients for at least 5 years would be useful.

\section{Acknowledgments}

We are grateful for the counsel of Professor Charles K. Job. We also acknowledge Mr P. Segar for his technical assistance and Mrs Reeny S. Charles for her secretarial help.

\section{References}

1 Treatment of paucibacillary leprosy. In chemotherapy of leprosy for control programmes. Report of a WHO group. WHO Tech Rep Ser, 1982; 675: 24.

2 WHO Expert Committee on leprosy. Sixth report, Tech Rep Ser, 1988; 768: 1.

3 Recent trends in chemotherapy of paucibacillary leprosy, ICMR Bulletin; 1990, 20: (6).

4 Ridley DS \& Jopling WH. Classification of leprosy according to immunity. A five group system. Int J Lepr, 1966; 34: 255-73.

5 Khanolkar VR. Method of taking biopsy tissue for histopathological examination. Lepr Rev, 1951; 22: 83-85.

6 Job CK and Chacko CJG. A modification of Fite's stain for demonstration of M. leprae in tissue sections. Int J Lepr, 1986; 58: 70-80.

7 Nilsen R, Mengistu G \& Reddy BB. The role of nerve biopsies in the diagnosis and management of leprosy. Lepr Rev, 1989; 60: 28-32. 
8 Sehgal VN, Rege VL \& Reys M. Correlation between clinical and histopathological classification in leprosy. Int J Lepr, 1977; 45: 278-280.

9 Srinivasan H, Rao KS \& Iyer CGS. Discrepancy in the histopathological features of leprosy lesions in the skin and peripheral nerve. Lepr Ind, 1982; 54: 275-282.

10 Katoch K, Ramu G, Ramanathan U \& Desikan KV. Comparison of three regimens containing rifampicin of paucibacillary leprosy patients. Int J Lepr, 1987; 62: 98-103.

11 Kumar B, Kaur S and Kaur I. Histological evaluation and follow up study of short term combination therapy for paucibacillary leprosy. Ind J Lepr, 1987; 59: 54-62.

12 Revankar CK, Ganapati R \& Naik DD. Multidrug therapy for paucibacillary leprosy. Experience in Bombay. Ind J Lepr, 1985; 57: 773-779.

13 Beovinger G, Ponnighaus JM and Fine PEM. Preliminary appraisal of WHO recommended multidrug regimen in paucibacillary patients in Malawi. Int J Lepr, 1988; 56: 408-417.

14 Ramu G. Duration of MDT for paucibacillary leprosy. Ind J Lepr, 1992; 64: 1-7.

15 Lowe J. The sulfone treatment of tuberculoid leprosy. Int J Lepr, 1950; 18: 457-468.

16 Turk JL \& MFR (1978) Leprosy. In: Immunological diseases 3rd ed., Boston, Samter M, Little Brown and Company, 627-638.

17 Job CK. Pathology of Leprosy. In Leprosy. Edited by Hastings RC. 2nd ed., Churchill Livingstone, (1994): 199200.

18 Katoch K, Ramanathan U, Natarajan M, Bagga AK, Bhatia AS, Saxena RK \& Ramu G. Relapses in paucibacillary patients after treatment with 3 short-term regimens containing rifampicin. Int J Lepr, 1989; 57: 458-464.

19 Pavithran K. Relapse of paucibacillary leprosy after short course multidrug therapy. Ind J Lepr, 1988; 60: 225229.

20 Ekambaram V \& Rao MK. Relapse rate in paucibacillary leprosy patients after multidrug therapy in North Arcot District. Ind J Lepr, 1991; 63: 34-42.

${ }^{21}$ Revankar CR, Karjivkar VG, Gurav VJ \& Ganapathi R. Clinical assessment of paucibacillary leprosy under multidrug therapy - three years follow up study. Ind J Lepr, 1989; 61: 355-359.

22 The leprosy Unit, WHO. Risk of relapse in leprosy. Ind J Lepr, 1955; 67: 13-26. 